\title{
16S rRNA gene sequencing reveals effects of photoperiod on cecal microbiota of broiler roosters
}

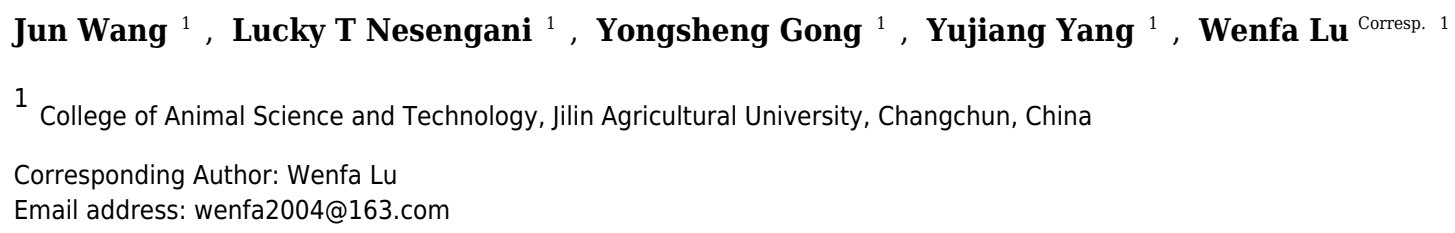

Photoperiod is an important factor in stimulating broiler performance in commercial poultry practice. However, the mechanism by which photoperiod affects the performance of broiler chickens has not been adequately explored. The current study evaluated the effects of 3 different photoperiod regimes (short day (LD) $=8 \mathrm{~h}$ light, control $(C T R)=12.5$ $\mathrm{h}$ light, and long day (SD) $=16 \mathrm{~h}$ light) on the cecal microbiota of broiler roosters by sequencing bacterial 16S rRNA genes. At the phylum level, the dominant bacteria were Firmicutes (CTR: 68\%, SD: 69\%, LD: 67\%) and Bacteroidetes (CTR: 25\%, SD: 26\%, and LD: $28 \%)$. There was a greater abundance of Proteobacteria $(p<0.01)$ and Cyanobacteria $(p<0.05)$ in chickens in the LD group than in those in the CTR group. A significantly greater abundance of Actinobacteria was observed in CTR chickens than in SD and LD chickens $(p<0.01)$. The abundance of Deferribacteres was significantly higher in LD chickens than in SD chickens $(p<0.01)$. Fusobacteria and Proteobacteria were more abundant in SD chickens than in CTR and LD chickens. The predicted functional properties indicate that cellular processes may be influenced by photoperiod. Conversely, carbohydrate metabolism was enhanced in CTR chickens as compared to that in SD and LD chickens. The current results indicate that different photoperiod regimes may influence the abundance of specific bacterial populations and then contribute to differences in the functional properties of gut microbiota of broiler roosters. 
$116 \mathrm{~S}$ rRNA gene sequencing reveals effects of photoperiod on cecal microbiota of broiler roosters

7 \# These authors contributed equally to this work

8

$9 *$ Corresponding author: Wenfa Lu,

10 E-mail: wenfa2004@163.com

11

12

13

14

15

16

17

18

19

20

21

22

23

24

25

26

27

28

29

30 


\section{ABSTRACT}

36

Photoperiod is an important factor in stimulating broiler performance in commercial poultry practice. However, the mechanism by which photoperiod affects the performance of broiler chickens has not been adequately explored. The current study evaluated the effects of 3 different photoperiod regimes (short day $(\mathrm{LD})=8 \mathrm{~h}$ light, control $(\mathrm{CTR})=12.5 \mathrm{~h}$ light, and long day (SD) $=16 \mathrm{~h}$ light) on the cecal microbiota of broiler roosters by sequencing bacterial 16S rRNA genes. At the phylum level, the dominant bacteria were Firmicutes (CTR: 68\%, SD: 69\%, LD: 67\%) and Bacteroidetes (CTR: 25\%, SD: 26\%, and LD: $28 \%$ ). There was a greater abundance of Proteobacteria $(p<0.01)$ and Cyanobacteria $(p<0.05)$ in chickens in the LD group than in those in the CTR group. A significantly greater abundance of Actinobacteria was observed in CTR chickens than in SD and LD chickens $(p<0.01)$. The abundance of Deferribacteres was significantly higher in LD chickens than in SD chickens $(\mathrm{p}<0.01)$. Fusobacteria and Proteobacteria were more abundant in SD chickens than in CTR and LD chickens. The predicted functional properties indicate that cellular processes may be influenced by photoperiod. Conversely, carbohydrate metabolism was enhanced in CTR chickens as compared to that in SD and LD chickens. The current results indicate that different photoperiod regimes may influence the abundance of specific bacterial populations and then contribute to differences in the functional properties of gut microbiota of broiler roosters.

\section{INTRODUCTION}

Photoperiod is defined as the relative amount of light per day to which an organism is exposed (Lee et al. 2017). This period of exposure to light can influence different aspects of physiology in avian as well as mammalian species, such as reproduction, behavior, and immune functions, to different magnitudes (Pittendrigh \& Daan 1976; Walton et al. 2011). Following photoperiod, animals tend to undergo a suite of adaptive responses by altering their physiology and reproductive state for survival (Walton et al. 2011). An increased photoperiod length has been reported to result in lower incidence of skeletal diseases and increase in weight gain with slower growth in broiler chickens (Classen et al. 1991). Decreasing or increasing photoperiod can also 
61 be used to reduce the early growth rate of broilers but allow them to compensate as they 62 approach market age (Downs et al. 2006). Photoperiod was also found to affect the physiology of 63 chickens; birds exposed to short days had a higher expression of gonadotropin-inhibitory 64 hormone expression compared to birds exposed to long days (Dixit et al. 2017). Furthermore, it 65 was reported that long photoperiods promote the development of the gonads in poultry (Kang \& Kuenzel 2015), although the exact mechanism underlying this effect is still unclear. Given the 67 many roles that photoperiod plays in various aspects of the physiology of avian and mammalian species, it is of scientific interest to evaluate its role in other inadequately explored aspects, such as the bacterial structure and functional properties of the gut microbiota.

Gut bacteria, which form part of the gut microbiota, have been shown to play important roles in digestion, metabolism, and health in avian species (Waite \& Taylor 2014; Waite \& Taylor 2015).

72 Gut microbiota have been widely reported to be affected by factors such as diet and age (Waite 73 \& Taylor 2014; Zhao et al. 2017; Zhu et al. 2017). However, other factors that may affect the 74 structure and functional properties of chicken gut microbiota, such as photoperiod, have yet to be 75 evaluated. Photoperiod may play a significant role in determining most physiological functions 76 by altering the gut microbiota. At present, there is a gap in the knowledge on the role of photoperiod in gut microbiota structure and function. The present study evaluated the effect of photoperiod on the abundance, diversity, and predicted functional properties of cecal microbiota in broiler roosters by sequencing the 16S rRNA gene.

MATERIALS AND METHODS

81

Ethical approval for the present study was obtained from the Ethical Committee of the Jilin Agricultural University, China.

\section{Photoperiod treatments}

44 One hundred and twenty AA+ Broilers (20 weeks of age, average weight: $2806 \mathrm{~g}$ ) were randomly divided into three groups $(\mathrm{n}=40)$ and subjected to different photoperiodic regimes for 5 weeks. Group I was designated the Control group (CTR; $12.5 \mathrm{~h}$ Light:11.5 h Dark, i.e., lights on at 08:00 a.m. and lights off at 08:30 p.m.), Group II the Long-day photoperiod group (SD; $16 \mathrm{~h}$ Light:8 h Dark, i.e., lights on at 04:00 p.m. and lights off at 08:00 a.m.), and Group III the Short- 
89 day photoperiod group (LD; $8 \mathrm{~h}$ Light:16 h Dark, i.e., lights on at 08:00 a.m. and lights off at 90 04:00 p.m.). A $60 \mathrm{~W}$ incandescent lamp with an illuminating intensity of 30 lux was used as the 91 source for artificial illumination and was positioned at the height of the head of standing birds. 92 All the broiler roosters were maintained in cages of equal size. Each rooster was fed $115 \mathrm{~g}$ of 93 commercial broiler diet per day for 20 weeks via restricted feeding before the experiment, and 94 then the amount of feed was increased by 5 g every week. In order to ensure that each rooster 95 was fed the same amount of diet, each rooster was kept in an individual cage. Water was 96 provided ad libitum during the whole experimental period.

\section{$97 \quad 2.2$ Sample collection}

98

All the roosters were slaughtered at about 25 weeks of age. Luminal cecum contents were collected from 7 randomly selected broilers from each group. All samples were harvested within $30 \mathrm{~min}$ after slaughter and immediately frozen in liquid nitrogen. The frozen luminal samples were stored in a freezer at $-80{ }^{\circ} \mathrm{C}$ until further use.

\section{DNA Extraction and 16S rRNA Amplification}

Samples were allowed to thaw at room temperature before DNA extraction. Total genomic DNA was extracted using the Fast DNA SPIN extraction kits (MP Biomedicals, Santa Ana, CA, USA), following the manufacturer's instructions. DNA concentration was evaluated by measuring optical density using Nano-Drop 2000 (Thermo Electron Corporation, USA) at wavelengths of 260 and $280 \mathrm{~nm}$. The integrity of the DNA extracts was assessed by electrophoresis on 1.0\% agarose gels. The V4-V5 regions of the bacterial 16S rRNA gene were amplified from the total microbial genomic DNA via PCR using the forward primer 515F (5'GTGCCAGCMGCCGCGGTAA-3') and the reverse primer 907R (5'CCGTCAATTCMTTTRAGTTT-3'). The amplification was carried out in $25 \mu \mathrm{L}$ reactions containing $5 \mu \mathrm{L}$ of Q5 reaction buffer $(5 \times), 5 \mu \mathrm{L}$ of Q5 High-Fidelity GC buffer $(5 \times), 0.25 \mu \mathrm{L}$ of Q5 High-Fidelity DNA Polymerase $(5 \mathrm{U} / \mu \mathrm{L}), 2 \mu \mathrm{L}$ of dNTPs $(2.5 \mathrm{mM}), 1 \mu \mathrm{L}$ each of the forward and reverse primer $(10 \mathrm{uM}), 2 \mu \mathrm{L}$ of DNA template, and $8.75 \mu \mathrm{L}$ of $\mathrm{ddH}_{2} \mathrm{O}$. PCR conditions were as follows: initial denaturation at $98{ }^{\circ} \mathrm{C}$ for $2 \mathrm{~min}$; followed by 25 cycles of denaturation at $98{ }^{\circ} \mathrm{C}$ for $15 \mathrm{~s}$, annealing at $55^{\circ} \mathrm{C}$ for $30 \mathrm{~s}$, and extension at $72{ }^{\circ} \mathrm{C}$ for $30 \mathrm{~s}$; and then a final extension at $72{ }^{\circ} \mathrm{C}$ for $5 \mathrm{~min}$. The PCR products were separated on $2 \%$ agarose gels 
118 and subsequently extracted from the gels. Samples with a bright band with a size between 200-

$119450 \mathrm{bp}$ were chosen for downstream experiments. PCR products were purified using a GeneJET

120 Gel Extraction Kit (Thermo Scientific, Waltham, USA). Products were quantified using a

121 PicoGreen dsDNA Assay Kit (Invitrogen, Carlsbad, CA, USA). After quantification, the 122 amplicons were pooled in equal amounts, and pair-end $2 \times 300-b p$ sequencing was performed 123 using the Illlumina MiSeq platform and a MiSeq Reagent Kit v3 at Shanghai Biotechnology Co., 124 Ltd (Shanghai, China).

\section{Bioinformatics and Statistical Analysis}

126 The quality control and analysis of the sequences were performed using the software 127 Quantitative Insights into Microbial Ecology (QIIME, v1.8.0) (Caporaso et al. 2010). The 128 paired-end reads from the DNA fragments were merged using FLASH (Magoc \& Salzberg 2011). 129 The UCLUST (Edgar 2010) clustering method was used to cluster operational taxonomic units 130 (OTUs) with $\geq 97 \%$ sequence identity. OTU classification was conducted by running a BLAST 131 search against the Greengenes Database (DeSantis et al. 2006) using the representative sequence 132 set as a query (Altschul et al. 1997). To minimize the difference in sequencing depth across 133 samples, an averaged, rounded, and rarefied OTU table was generated by averaging 100 evenly 134 re-sampled OTU subsets under the $90 \%$ of the minimum sequencing depth. These were then 135 used for further analysis.

136 Bioinformatics and statistical analyses were performed using the QIIME and R packages (v3.2.0). 137 The alpha-diversity indices (Chao1, ACE metric, Shannon diversity index, and Simpson index) 138 were calculated using the QIIME software to establish the abundance and diversity of the 139 sequences. Beta-diversity was determined using unweighted UniFrac distance metrics to evaluate 140 the structure and distribution of the microbial genetic communities among the samples 141 (Lozupone \& Knight 2005; Lozupone et al. 2007). Differences in the Unifrac distances for 142 pairwise comparisons among groups were calculated using Student's $t$-test and the Monte Carlo 143 permutation test with 1000 permutations. Significance was assigned when $\mathrm{p}<0.05$ and $\mathrm{p}<0.01$.

144 The differences and similarities between the compared groups were evaluated using ANOSIM 145 (analysis of similarities) in the R package "vegan." A Venn diagram was generated using the R 146 package "VennDiagram" to visualize the shared and unique OTUs among samples or groups.

147 Functional genes were predicted using PICRUSt (phylogenetic investigation of communities by 
148 reconstruction of unobserved states) using high-quality sequences as the input (Langille et al. 149 2013).

\section{RESULTS}

\section{Sequencing overview}

152 A total of 21 samples were obtained from three groups ( $\mathrm{n}=7$ per group) of broiler roosters and 153 subsequently sequenced to generate V4-V5 16S rRNA gene profiles. A total of 398445, 328235, 154 and 375402 sequences were obtained for the CTR, SD, and LD groups, respectively. There was 155 an average of 56920,46890 , and 53628 reads per sample in the CTR, SD, and LD groups, 156 respectively.

157 Validation and structure determination of the sequences

158 The variation in data distribution between the groups was analyzed using ANOSIM, which 159 indicated a significant difference $(p<0.01)$ between the three groups under unweighted Unifrac.

160 The alpha-diversity indices (chao1, Simpson and Shannon index) are reported in Table 1, there 161 was a significant difference between CTR and SD groups when comparing a chao1 indices mean. 162 The results of the beta diversity analysis and the PLS-discriminant analysis are shown in Fig. 1 163 and 2, respectively. Samples from LD and SD indicated to be clustered similar where the CTR 164 samples were different in NMDS analysis. The PLS-discriminant analysis indicated that the two 165 groups are different with the exception of one sample from LD which was clustered with SD.

166 Abundance and Significant difference between the three groups at the Phylum level

167 The most abundant bacteria at the phylum level were Firmicutes, with abundances of 68\%, 69\%, 168 and $67 \%$ in the CTR, SD, and LD groups, respectively, followed by Bacteroidetes with 169 abundances at 25\%,26\%, and $28 \%$ in the CTR, SD, and LD groups, respectively (Fig. 3). The 170 other bacterial phyla had abundances lower than 3\% in all groups at varying magnitudes. As 171 shown in Fig. 2, Proteobacteria $(p<0.01)$ and Cyanobacteria $(\mathrm{p}<0.05)$ were more abundant in 172 LD chickens than in CTR chickens, while Actinobacteria was more abundant in chicken from 173 the CTR group than in those from the LD group $(p<0.01)$. Between the CTR and SD groups, 174 there was a significant difference in the abundance of Actinobacteria, which was more abundant 175 in the CTR group than in the SD group $(p<0.01)$. Deferribacteres was more abundant in LD 176 roosters than in SD roosters $(p<0.05)$. Fusobacteria and Proteobacteria were significantly more 
177 abundant $(p<0.01)$ in chickens from the SD group than in those from the CTR and LD groups, as 178 indicated in Fig. 4.

\section{Abundance and Significance difference between the three groups at the Genus level}

180 The most abundant bacteria at the genus level were Bacteroides, with $15 \%, 13 \%$, and $15 \%$ 181 abundances in the CTR, SD, and LD groups, respectively. This was followed by unclassified 182 Ruminococcaceae at 13\%, 14\%, and 14\% abundances in the CTR, SD, and LD groups, 183 respectively (Fig. 5). Other abundant genera included Ruminococcus (CTR: 14\%, SD: 9\%, LD: 184 10\%), unclassified Clostridiales (CTR: 9\%, SD: 11\%, LD: 12\%), and Faecalibacterium (CTR: $1858 \%$, SD: $10 \%$, LD: $8 \%$ ). Ten genera were significantly $(p<0.01)$ different in abundance between 186 the CTR and SD groups, 7 between the CTR and LD groups, and 5 between the SD and LD 187 groups. Also importantly the genus Aeriscardovia was significantly more abundant $(p<0.01)$ in 188 the LD than in the SD and CTR groups (Fig. 6). Interestingly, Megamonas, Ochrobactrum, and 189 Selenomonas were significantly more abundant $(p<0.01)$ in the CTR group than in the other two 190 groups. Aeriscardovia, Delftia, and Rikenella were significantly more abundant $(p<0.01)$ in the

191 LD group than in the CTR and SD groups. Lactococcus and Fusobacterium were significantly 192 more abundant $(p<0.01)$ in the SD group than in the other two groups (Fig. 6). A heat map 193 indicating significantly expressed genera is shown in Fig. 7.

\section{Differences in Predicted Functional properties between the three groups}

195 The differences in the effect of photoperiod on the functional properties across the three groups 196 were further evaluated. Moderate differences were observed in cellular processes, particularly, in 197 cell motility (Fig. 8). Cell motility was relatively low in samples from the CTR group compared 198 to the motility in samples from the SD and LD groups. However, there were no notable 199 differences in other functions such as transport and catabolism, cell growth and death, and cell 200 communication across the groups. Analysis of the metabolism of the samples showed that 201 carbohydrate metabolism was enhanced in CTR samples as compared to those in the SD and LD 202 samples. Other functions did not exhibit any differences across the three tested groups (Fig. 9). 203 Similar results were observed in other functional processes, such as genetic information 204 processes and environmental information processes (results not shown).

205 DISCUSSION 
206 Photoperiodism provides animals with the ability to change many physiological aspects and, 207 consequently, adapting their body to the environment depending on the duration of light 208 exposure (Bailey et al. 2010). The current study evaluated the structure and functional properties 209 of the cecal microbiota of roosters that were subjected to three different photoperiodic regimes. 210 Our data indicates that the length of time of light exposure may affect the abundance of specific 211 bacteria in the cecum, leading to possible changes in functional properties. These changes may 212 range across a variety of aspects the underlying mechanism of which has not been sufficiently 213 explored.

214 To the best of our knowledge, there are few reports on the effects of photoperiod on gut 215 microbiota in chickens and even in other species. Recently, a study demonstrated the role of 216 photoperiod in changing gut microbiota. It indicated that different photoperiodic regimes $(8 \mathrm{~h}$ 217 dark $/ 16 \mathrm{~h}$ light, $12 \mathrm{~h}$ dark/12 h light, and $16 \mathrm{~h}$ dark $/ 8 \mathrm{~h}$ light cycles) could shape the gut 218 microbiota of mice and thereby affect host radio sensitivity (Cui et al. 2016). The results of the 219 present study are in general agreement with the findings of previous studies. Previously, it was 220 reported that Firmicutes and Bacteroidetes dominate the broiler gut microbiota (Cui et al. 2017; 221 Oh et al. 2017a; Oh et al. 2017b; Zhou et al. 2017), although effects of photoperiod on their 222 abundances were not demonstrated. It was noted that Megamonas was significantly more 223 abundant in CTR group as compared to LD and SD groups. It is of interest to note that 224 Megamonas has been previously reported to play a significant role in fermenting glucose into 225 acetate and propionate, which is pivotal for health benefits in a few species such as humans and 226 ducks (Chevrot et al. 2008; Sakon et al. 2008; Zhang et al. 2013). These findings may indicate a 227 new platform for manipulating acetate and propionate in broiler roosters by varying photoperiod 228 regimes. Short term (LD) exposure to light has also been indicated to significantly increase the 229 abundance of the novel genus Aeriscardovia (Simpson et al. 2004) and of the gram-negative 230 bacteria Delftia, which has been reported to be associated with infectious diseases (Bilgin et al. 231 2015; Calzada et al. 2015). Long term (SD) exposure to light significantly increases the 232 abundance of gram-positive Lactococcus, which has been reported to have potential for use in 233 preventing infectious diseases (Hanniffy et al. 2007). The gram-negative genus Fusobacterium, 234 which has been reported to be associated with infections in humans (Kostic et al. 2013), was also 235 significantly more abundant under an SD regime. Our results seem to indicate that short term 236 (LD) photoperiod ( $8 \mathrm{~h}$ light) could increase the abundance of bacterial genera associated with 
237 infectious diseases in the rooster gut, while long term photoperiod ( $16 \mathrm{~h} \mathrm{light)} \mathrm{could} \mathrm{increase} \mathrm{the}$

238 abundance of bacterial genera associated with preventing infectious diseases. However, this 239 deduction needs to be verified by more extensive scientific investigation. These results are of 240 importance to prompt more studies on the role that photoperiod may play with regards to 241 physiology in animals. It is of note that different results may arise due to differences in time of 242 exposure, light intensity, animal and growth stages, and other factors employed in the study.

243 The effect on several functional properties of the roosters may be attributed to increases in the 244 abundance of specific bacteria caused by light exposure duration. Analysis of predicted 245 functional properties in the present study indicated that metabolism may be influenced by 246 photoperiod. Carbohydrate metabolism was enhanced in the CTR group, as compared to the SD 247 and LD groups. Previous studies have demonstrated that gut microbiota plays an important role 248 in the life activities of chickens (Waite \& Taylor 2014). The present study is limited by the fact 249 that the change in gut microbiota was not correlated with performance (e.g., testis development or body weight). Further studies are suggested to investigate the effects of photoperiod on gut microbiota and their relationship with growth or reproduction performance.

\section{CONCLUSIONS}

253

254

255

256

257

258

259

260

261

262

263

264

265

266

267

268

269

270

271

Our results indicate that photoperiod may affect the abundance of specific bacteria in the gut and thereby contribute to differences in the functional properties of the gut microbiota in broiler roosters.

\section{REFERENCES}

Altschul SF, Madden TL, Schaffer AA, Zhang JH, Zhang Z, Miller W, and Lipman DJ. 1997. Gapped BLAST and PSI-BLAST: a new generation of protein database search programs. Nucleic Acids Research 25:3389-3402. 10.1093/nar/25.17.3389

Bailey MT, Walton JC, Dowd SE, Weil ZM, and Nelson RJ. 2010. Photoperiod modulates gut bacteria composition in male Siberian hamsters (Phodopus sungorus). Brain Behav Immun 24:577-584. 10.1016/j.bbi.2009.12.010

Bilgin H, Sarmis A, Tigen E, Soyletir G, and Mulazimoglu L. 2015. Delftia acidovorans: A rare pathogen in immunocompetent and immunocompromised patients. Can J Infect Dis Med Microbio/ 26:277-279.

Calzada M, Roig M, Martinez-Toldos MC, and Segovia M. 2015. [Urinary tract infection associated with Delftia acidovorans]. Rev Esp Quimioter 28:326-327.

Caporaso JG, Kuczynski J, Stombaugh J, Bittinger K, Bushman FD, Costello EK, Fierer N, Pena AG, Goodrich JK, Gordon JI, Huttley GA, Kelley ST, Knights D, Koenig JE, Ley RE, Lozupone CA, McDonald D, Muegge BD, Pirrung M, Reeder J, Sevinsky JR, Turnbaugh PJ, Walters WA, Widmann J, Yatsunenko T, Zaneveld J, and Knight R. 2010. QIIME allows 
272

273

274

275

276

277

278

279

280

281

282

283

284

285

286

287

288

289

290

291

292

293

294

295

296

297

298

299

300

301

302

303

304

305

306

307

308

309

310

311

312

313

314

315

316

317

318

analysis of high-throughput community sequencing data. Nat Methods 7:335-336. 10.1038/nmeth.f.303

Chevrot R, Carlotti A, Sopena V, Marchand P, and Rosenfeld E. 2008. Megamonas rupellensis sp. nov., an anaerobe isolated from the caecum of a duck. Int J Syst Evol Microbiol 58:2921-2924. 10.1099/ijs.0.2008/001297-0

Classen HL, Riddell C, and Robinson FE. 1991. Effects of increasing photoperiod length on performance and health of broiler chickens. British Poultry Science 32:21-29. $10.1080 / 00071669108417324$

Cui M, Xiao H, Luo D, Zhang X, Zhao S, Zheng Q, Li Y, Zhao Y, Dong J, Li H, Wang H, and Fan S. 2016. Circadian Rhythm Shapes the Gut Microbiota Affecting Host Radiosensitivity. International Journal of Molecular Sciences 17:1786. 10.3390/ijms17111786

Cui Y, Wang Q, Liu S, Sun R, Zhou Y, and Li Y. 2017. Age-Related Variations in Intestinal Microflora of Free-Range and Caged Hens. Front Microbiol 8:1310. 10.3389/fmicb.2017.01310

DeSantis TZ, Hugenholtz P, Larsen N, Rojas M, Brodie EL, Keller K, Huber T, Dalevi D, Hu P, and Andersen GL. 2006. Greengenes, a chimera-checked 16S rRNA gene database and workbench compatible with ARB. Applied and Environmental Microbiology 72:5069-5072. 10.1128/aem.03006-05

Dixit AS, Singh NS, and Byrsat S. 2017. Role of GnIH in photoperiodic regulation of seasonal reproduction in the Eurasian tree sparrow. The Journal of Experimental Biology 220:3742.

Downs KM, Lien RJ, Hess JB, Bilgili SF, and Dozier IWA. 2006. The Effects of Photoperiod Length, Light Intensity, and Feed Energy on Growth Responses and Meat Yield of Broilers. The Journal of Applied Poultry Research 15:406-416. 10.1093/japr/15.3.406

Edgar RC. 2010. Search and clustering orders of magnitude faster than BLAST. Bioinformatics 26:2460-2461. 10.1093/bioinformatics/btq461

Hanniffy SB, Carter AT, Hitchin E, and Wells JM. 2007. Mucosal Delivery of a Pneumococcal Vaccine Using Lactococcus lactis Affords Protection against Respiratory Infection. The Journal of Infectious Diseases 195:185-193.

Kang SW, and Kuenzel WJ. 2015. Deep-brain photoreceptors (DBPs) involved in the photoperiodic gonadal response in an avian species, Gallus gallus. Gen Comp Endocrinol 211:106-113. 10.1016/j.ygcen.2014.11.020

Kostic AD, Chun E, Robertson L, Glickman JN, Gallini CA, Michaud M, Clancy TE, Chung DC, Lochhead P, Hold GL, El-Omar EM, Brenner D, Fuchs CS, Meyerson M, and Garrett WS. 2013. Fusobacterium nucleatum potentiates intestinal tumorigenesis and modulates the tumor immune microenvironment. Cell host \& microbe 14:207-215. 10.1016/j.chom.2013.07.007

Langille MGI, Zaneveld J, Caporaso JG, McDonald D, Knights D, Reyes JA, Clemente JC, Burkepile DE, Thurber RLV, Knight R, Beiko RG, and Huttenhower C. 2013. Predictive functional profiling of microbial communities using $16 \mathrm{~S}$ rRNA marker gene sequences. Nature Biotechnology 31:814-+. 10.1038/nbt.2676

Lee C-H, Park Y-J, and Lee Y-D. 2017. Effects of Photoperiod Manipulation on Gonadal Activity of the Damselfish, Chromis notata. Development \& Reproduction 21:223-228. 10.12717/DR.2017.21.2.223

Lozupone C, and Knight R. 2005. UniFrac: a new phylogenetic method for comparing microbial communities. Applied and Environmental Microbiology 71:8228-8235. 10.1128/aem.71.12.8228-8235.2005 
319

320

321

322

323

324

325

326

327

328

329

330

331

332

333

334

335

336

337

338

339

340

341

342

343

344

345

346

347

348

349

350

351

352

353

354

355

356

357

358

359

360

361

362

363
Lozupone CA, Hamady M, Kelley ST, and Knight R. 2007. Quantitative and qualitative beta diversity measures lead to different insights into factors that structure microbial communities. Applied and Environmental Microbiology 73:1576-1585. 10.1128/aem.01996-06

Magoc T, and Salzberg SL. 2011. FLASH: fast length adjustment of short reads to improve genome assemblies. Bioinformatics 27:2957-2963. 10.1093/bioinformatics/btr507

Oh JK, Pajarillo EAB, Chae JP, Kim IH, Yang DS, and Kang D-K. 2017a. Effects of Bacillus subtilis CSL2 on the composition and functional diversity of the faecal microbiota of broiler chickens challenged with Salmonella Gallinarum. Journal of Animal Science and Biotechnology 8:1. 10.1186/s40104-016-0130-8

Oh JK, Pajarillo EAB, Chae JP, Kim IH, Yang DS, and Kang DK. 2017b. Effects of Bacillus subtilis CSL2 on the composition and functional diversity of the faecal microbiota of broiler chickens challenged with Salmonella Gallinarum. J Anim Sci Biotechno/ 8:1. 10.1186/s40104-016-0130-8

Pittendrigh CS, and Daan S. 1976. A functional analysis of circadian pacemakers in nocturnal rodents. Journal of comparative physiology 106:333-355. 10.1007/BF01417860

Sakon H, Nagai F, Morotomi M, and Tanaka R. 2008. Sutterella parvirubra sp. nov. and Megamonas funiformis sp. nov., isolated from human faeces. Int J Syst Evol Microbiol 58:970-975. 10.1099/ijs.0.65456-0

Simpson PJ, Ross RP, Fitzgerald GF, and Stanton C. 2004. Bifidobacterium psychraerophilum sp. nov. and Aeriscardovia aeriphila gen. nov., sp. nov., isolated from a porcine caecum. Int J Syst Evol Microbio/ 54:401-406. 10.1099/ijs.0.02667-0

Waite DW, and Taylor MW. 2014. Characterizing the avian gut microbiota: membership, driving influences, and potential function. Front Microbio/ 5:223. 10.3389/fmicb.2014.00223

Waite DW, and Taylor MW. 2015. Exploring the avian gut microbiota: current trends and future directions. Front Microbio/ 6:673. 10.3389/fmicb.2015.00673

Walton JC, Weil ZM, and Nelson RJ. 2011. Influence of Photoperiod on Hormones, Behavior, and Immune Function. Frontiers in neuroendocrinology 32:303-319. 10.1016/j.yfrne.2010.12.003

Zhang X, Shen D, Fang Z, Jie Z, Qiu X, Zhang C, Chen Y, and Ji L. 2013. Human Gut Microbiota Changes Reveal the Progression of Glucose Intolerance. PLoS One 8:e71108. 10.1371/journal.pone.0071108

Zhao F, Huang Z, Zhou G, Li H, Xu X, and Li C. 2017. Dietary Proteins Rapidly Altered the Microbial Composition in Rat Caecum. Curr Microbiol. 10.1007/s00284-017-1339-2

Zhou Z, Nie K, Huang Q, Li K, Sun Y, Zhou R, Wang Z, and Hu S. 2017. Changes of cecal microflora in chickens following Eimeria tenella challenge and regulating effect of coated sodium butyrate. Exp Parasitol 177:73-81. 10.1016/j.exppara.2017.04.007

Zhu Y, Shi X, Lin X, Ye K, Xu X, Li C, and Zhou G. 2017. Beef, Chicken, and Soy Proteins in Diets Induce Different Gut Microbiota and Metabolites in Rats. Front Microbio/ 8:1395. 10.3389/fmicb.2017.01395 
364

365

366

367

368

369

370

371

PeerJ reviewing PDF | (2017:10:21463:2:0:NEW 22 Jan 2018) 


\section{Table $\mathbf{1}$ (on next page)}

The average alpha-diversity indexes (chaol, Simpson and Shannon index) of the data distribution

Numbers with asterisks are significantly different ( $p$ value $<0.05$ ). 


\begin{tabular}{ccccccc}
\hline Group & \multicolumn{2}{c}{ Chao1 } & \multicolumn{2}{c}{ Simpson } & \multicolumn{2}{c}{ Shannon } \\
\hline & Mean & STD & Mean & STD & Mean & STD \\
& & & & & & \\
CTR & $1799.593 *$ & 256.3406 & 0.972857 & 0.00488 & 7.411429 & 0.281569 \\
& & & & & & \\
SD & $1461.779 *$ & 310.5823 & 0.977143 & 0.00488 & 7.525714 & 0.227146 \\
& & & & & & \\
LD & 1729.097 & 224.2392 & 0.975714 & 0.007868 & 7.607143 & 0.223958 \\
\hline
\end{tabular}




\section{Figure 1}

The beta diversity results of NMDS indicating the data distribution between the groups

LD refers to the $8 \mathrm{~h}$ light group samples, CTR refers to the $12.5 \mathrm{~h}$ light and SD refers to the $16 \mathrm{~h}$ light group samples respectively.

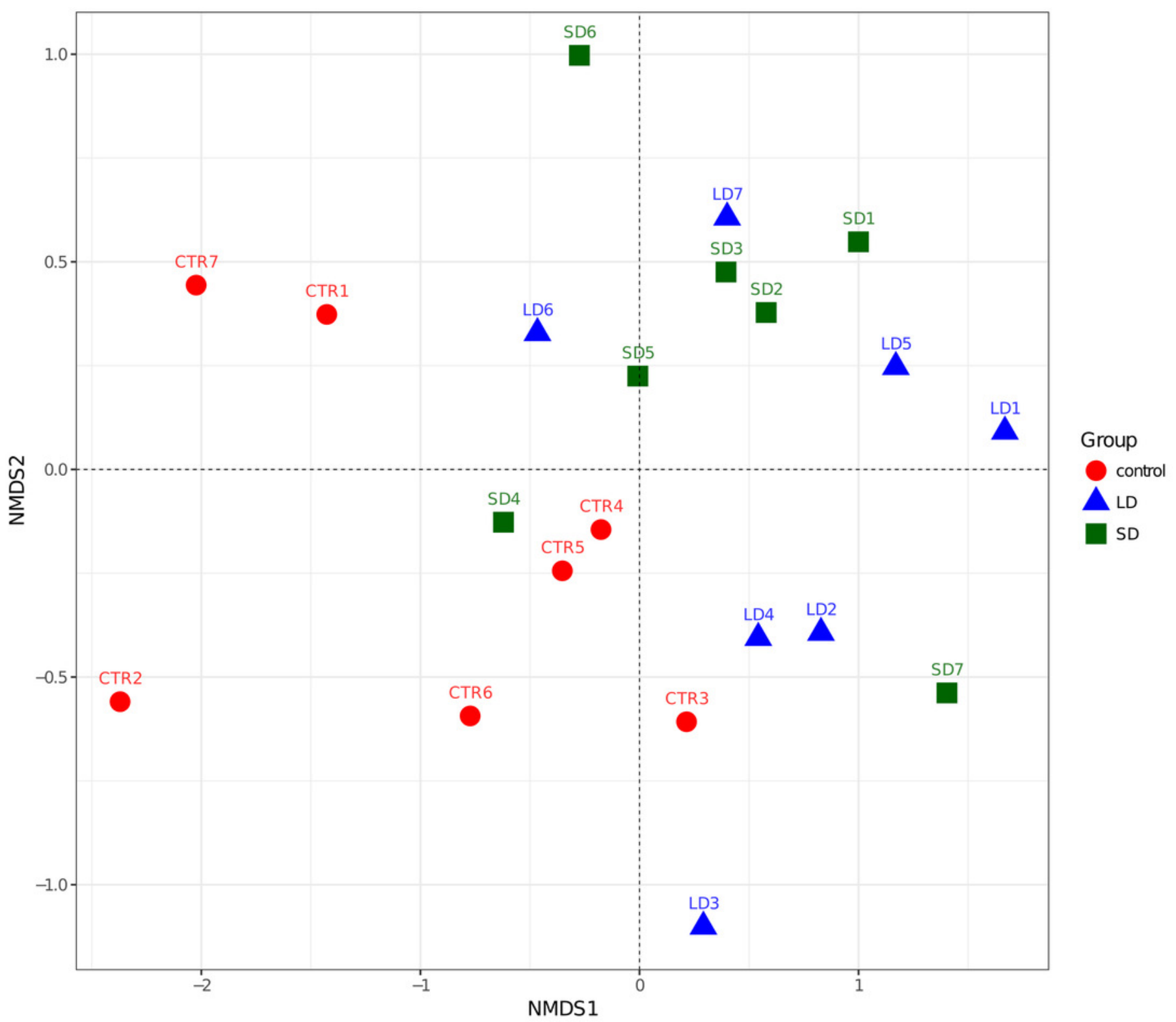


Figure 2

The PLS-discriminant analysis

LD refers to the $8 \mathrm{~h}$ light group samples, CTR refers to the $12.5 \mathrm{~h}$ light and SD refers to the $16 \mathrm{~h}$ light group samples respectively.

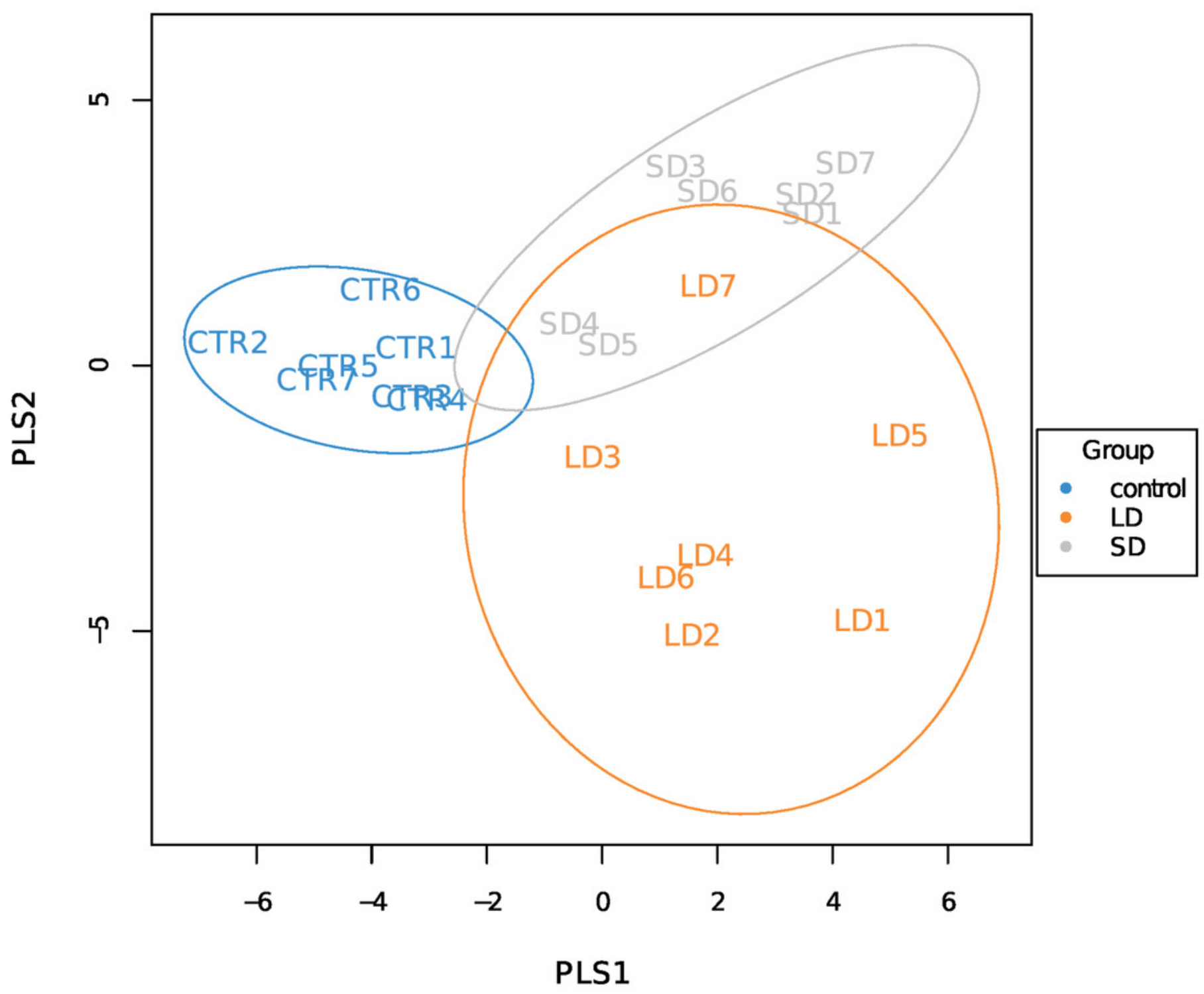


Figure 3

Taxonomic profiles of the microbial communities at the phylum level

LD refers to the $8 \mathrm{~h}$ light group, CTR refers to the $12.5 \mathrm{~h}$ light and SD refers to the $16 \mathrm{~h}$ light group. Samples are presented along with the horizontal axis and relative abundance at the vertical axis.

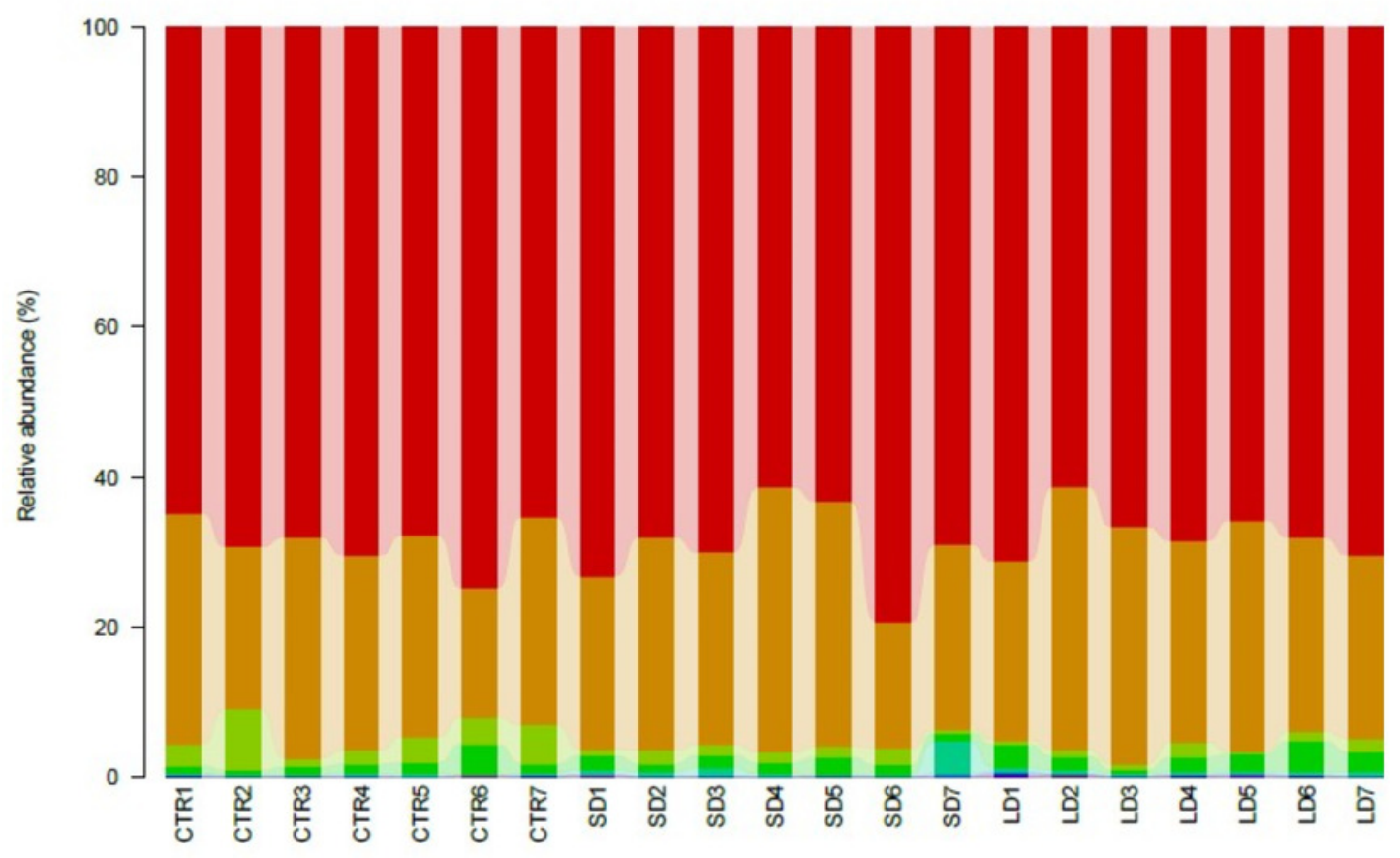

Firmicutes

Bacteroidetes

Actinobacteria

Tenericutes

Proteobacteria

Cyanobacteria

Synergistetes

TM7

Verrucomicrobia

Deferribacteres

Elusimicrobia

Fusobacteria

Others 
Figure 4

Taxonomic profiles of the notable significant different bacterium at the phylum level

Samples/groups are as previously explained. Different uppercase and lowercase letters indicate significance of difference at $p<0.01$ and $p<0.05$, respectively. Same letters indicate no significant difference.

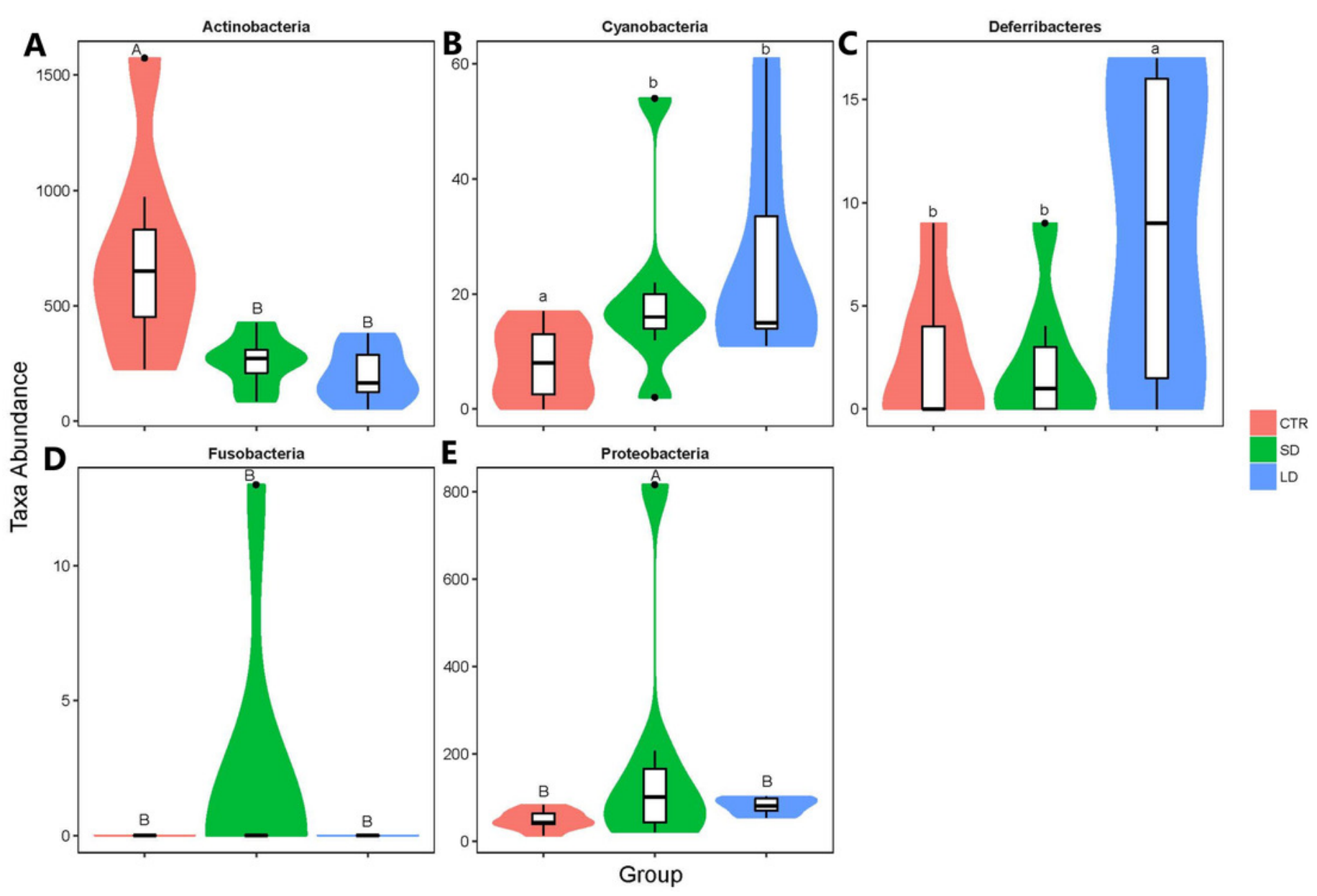


Figure 5

Taxonomic profiles of the microbial communities at the genus level

Samples/groups are as previously explained.

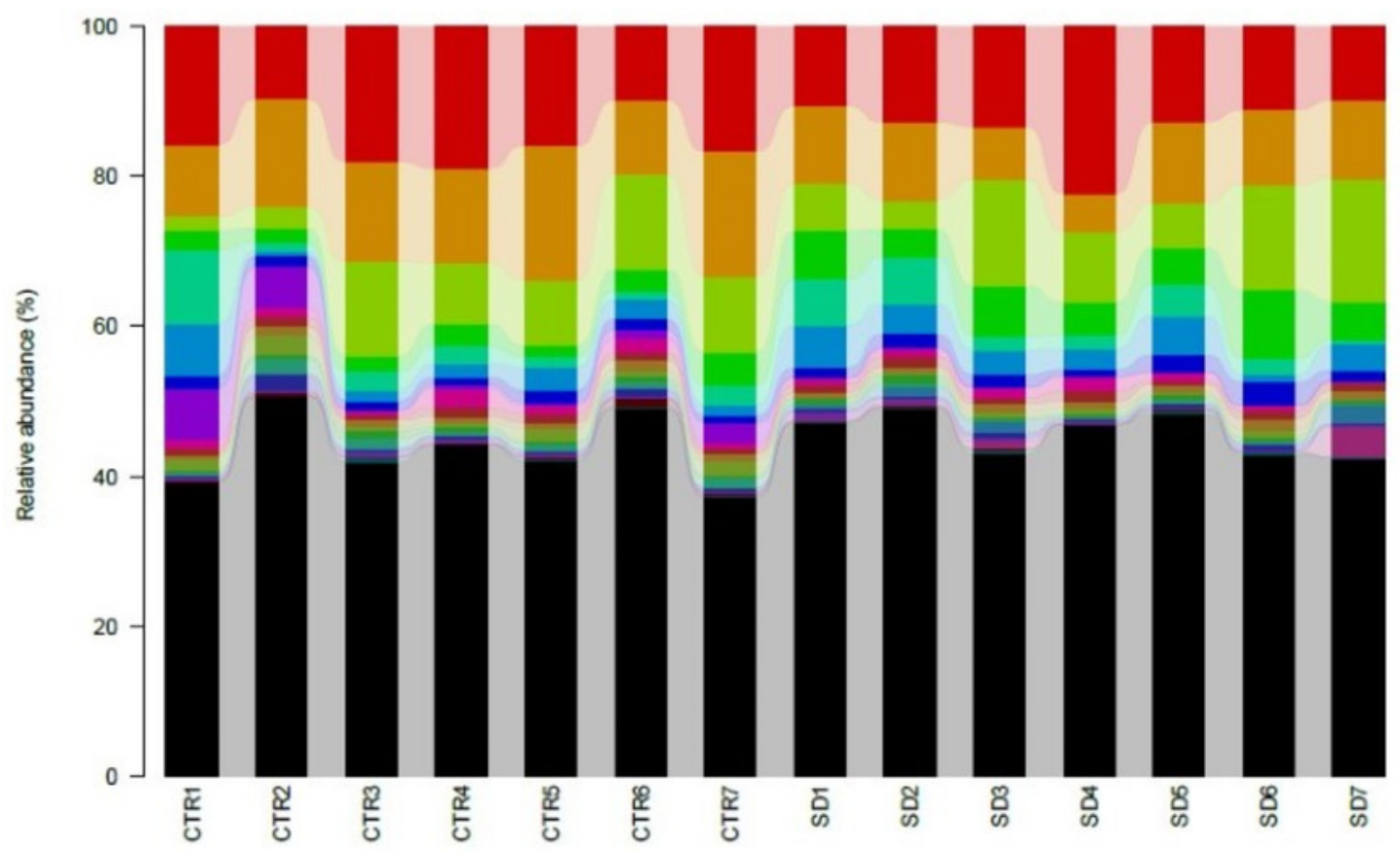

Bacteroides

[Ruminococcus]

Faecalibacterium

Lactobacillus

Streptococcus

Alistipes

Oscillospira

Megamonas

Coprobacillus

Ruminococcus

Dorea

Bifidobacterium

Coprococcus

Peptococcus

Clostridium

Blautia

cc_115

Desulfovibrio

Collinsella

Parabacteroides

Others 
Figure 6

Taxonomic profiles of the notable significant different bacterium at the genus level

Samples/groups are as previously explained. Different uppercase and lowercase letters indicate significance of difference at $p<0.01$ and $p<0.05$, respectively. Same letters indicate no significant difference.

A
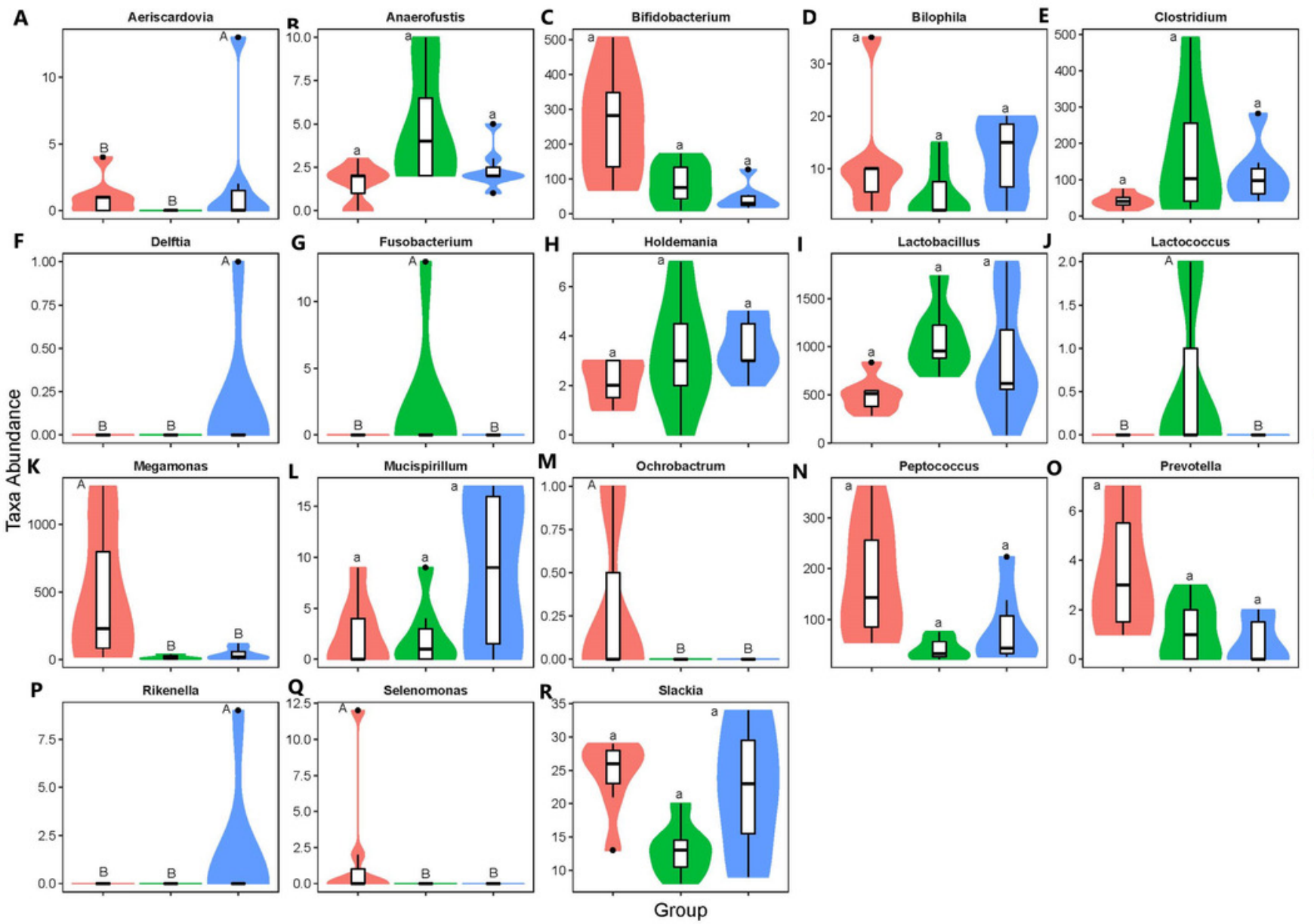


\section{Figure 7}

Heatmap showing the genera with significant differences of relative abundances amongst the 3 groups

Heatmap is color-coded based on the scale of -4 to 4 .

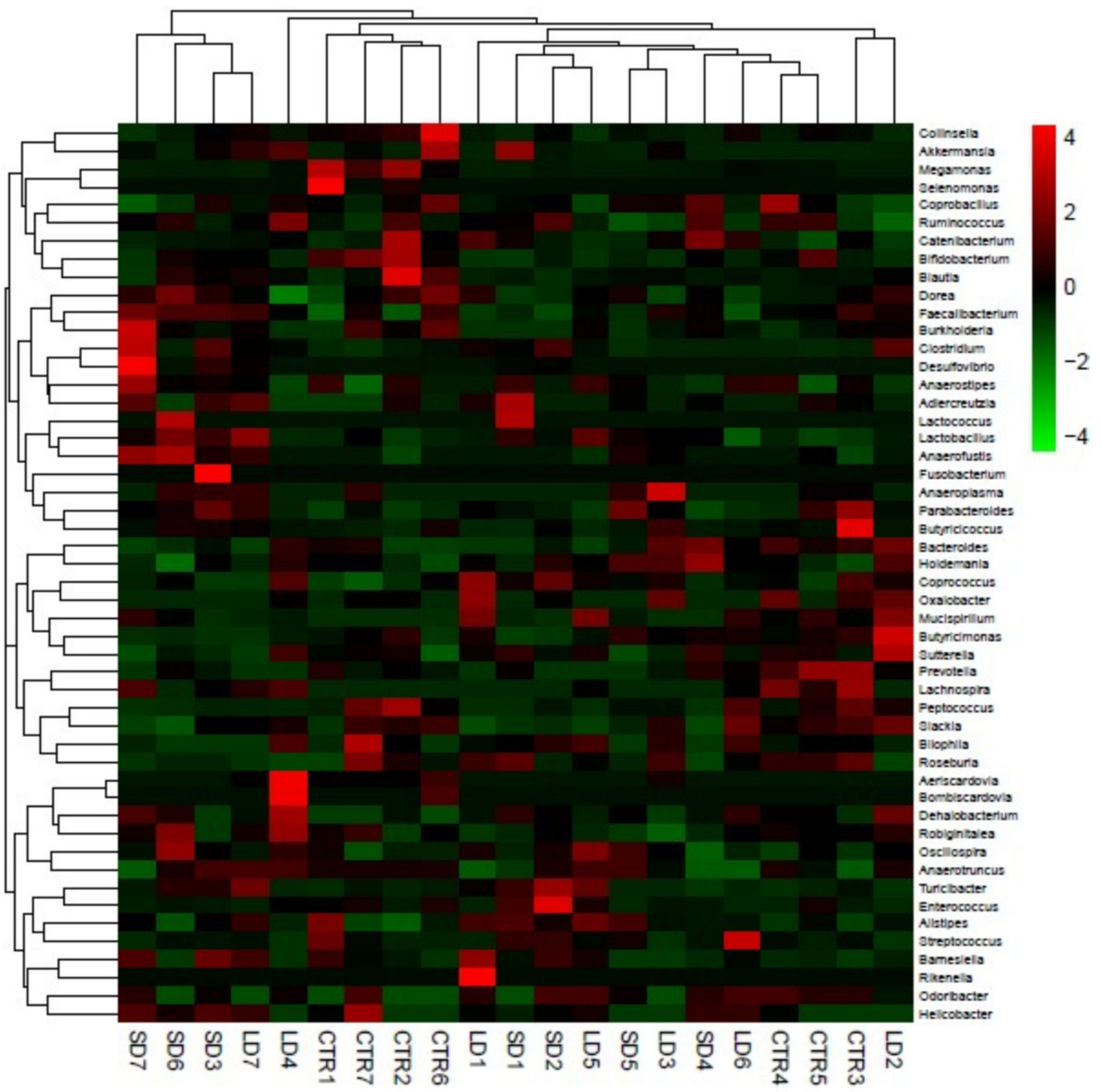


Figure 8

Representing the functional differences at the cellular processes

Samples/groups are as previously explained.

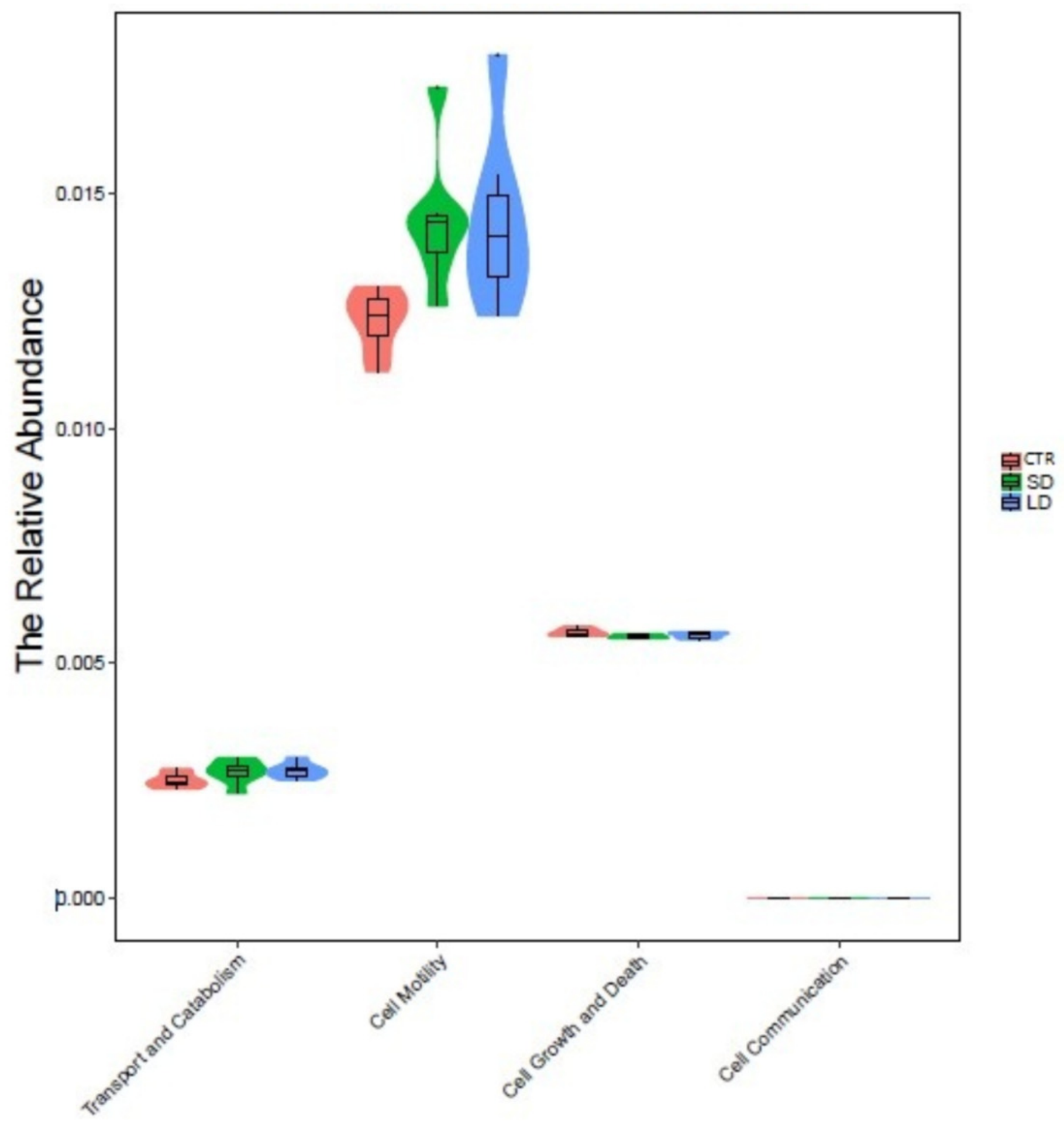


Figure 9

Representing the functional differences at the metabolism level between the 3 groups

Samples/groups are as previously explained.

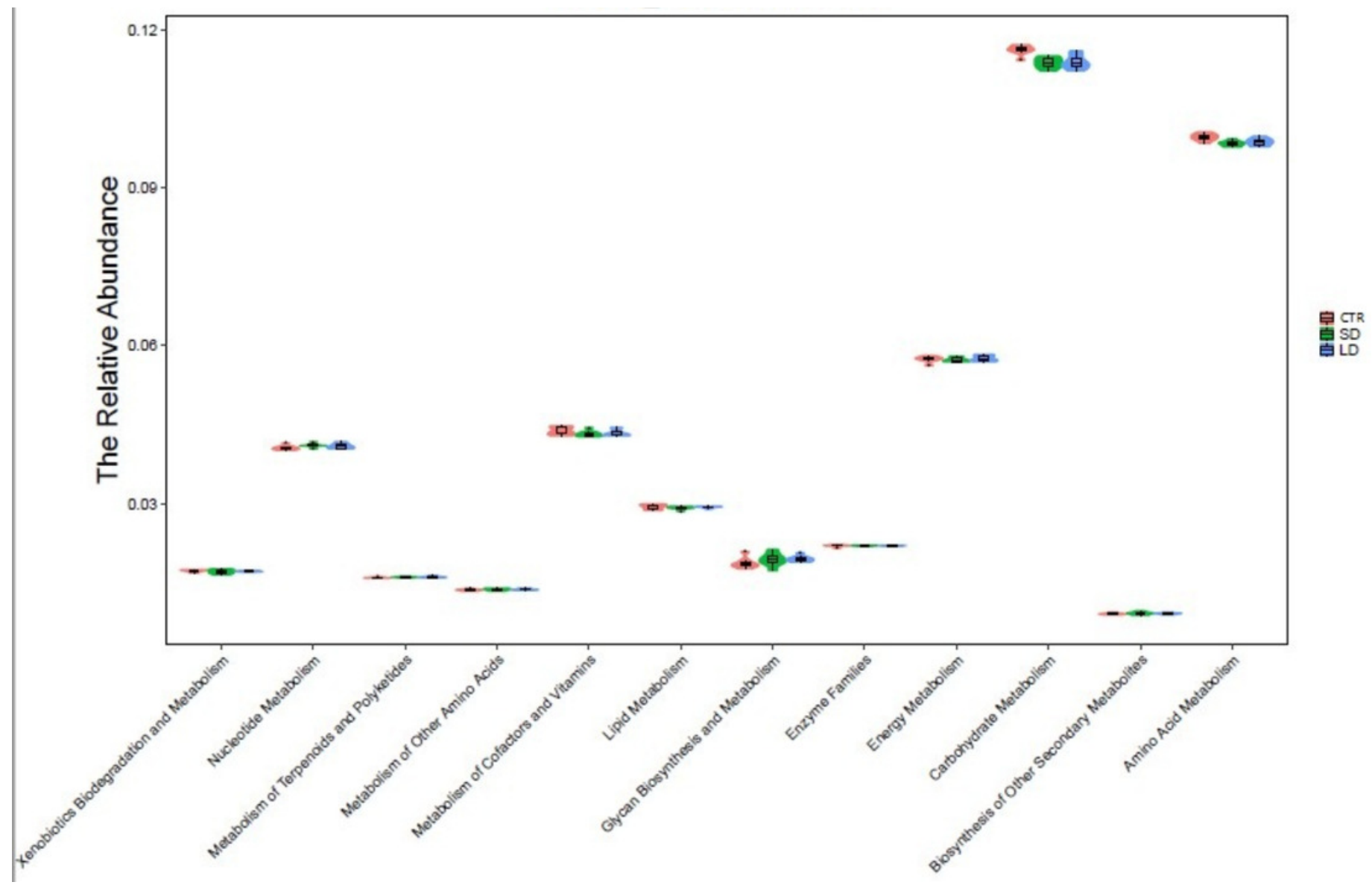

